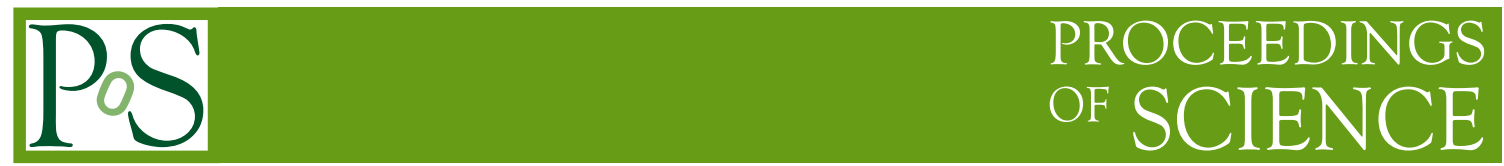

\title{
Precision Higgs physics at the ILC, and its impact on detector design
}

\section{Daniel Jeans $^{a, 1, *}$}

${ }^{a}$ High Energy Accelerator Research Organization (KEK),

Tsukuba, Japan

E-mail: daniel.jeans@kek.jp

We summarise the potential of the International Linear Collider to measure the Higgs boson's properties. These measurements profit from both the ILC environment and suitably designed detectors. We discuss the detector requirements to optimally extract information from collisions provided at the ILC.

40th International Conference on High Energy physics - ICHEP2020

July 28 - August 6, 2020

Prague, Czech Republic (virtual meeting)

${ }^{1}$ On behalf of the Linear Collider Collaboration.

* Speaker 


\section{Introduction}

Properties of the Higgs sector are expected to bear the marks of physics at higher scales, beyond the current Standard Model (SM) of particle physics. Couplings between the Higgs and other SM particles are typically varied by of order a few percent if new physics lies at the TeV-scale. The enhancements and suppression of different Higgs couplings depends on both the type of new physics lying beyond the SM, and on its energy scale. Measurement of these couplings at the percent-level is needed to detect such deviations and to distinguish different models of the new physics and deduce their energy scale.

The main motivation for the International Linear Collider (ILC) is to make such precision measurements of the Higgs boson's characteristics [1]. Electron-positron collisions can produce Higgs bosons via several processes, as shown in fig. 1. The Higgs-strahlung process reaches peak cross-section at around $250 \mathrm{GeV}$, while Higgs production via WW and $\mathrm{ZZ}$ fusion become more important at higher energies. From around $500 \mathrm{GeV}$, additional processes become significant: Higgs produced together with a pair of top quarks, allowing direct measurement of the top quark's Yukawa coupling; and double Higgs boson production, which provides access to the Higgs self-coupling.

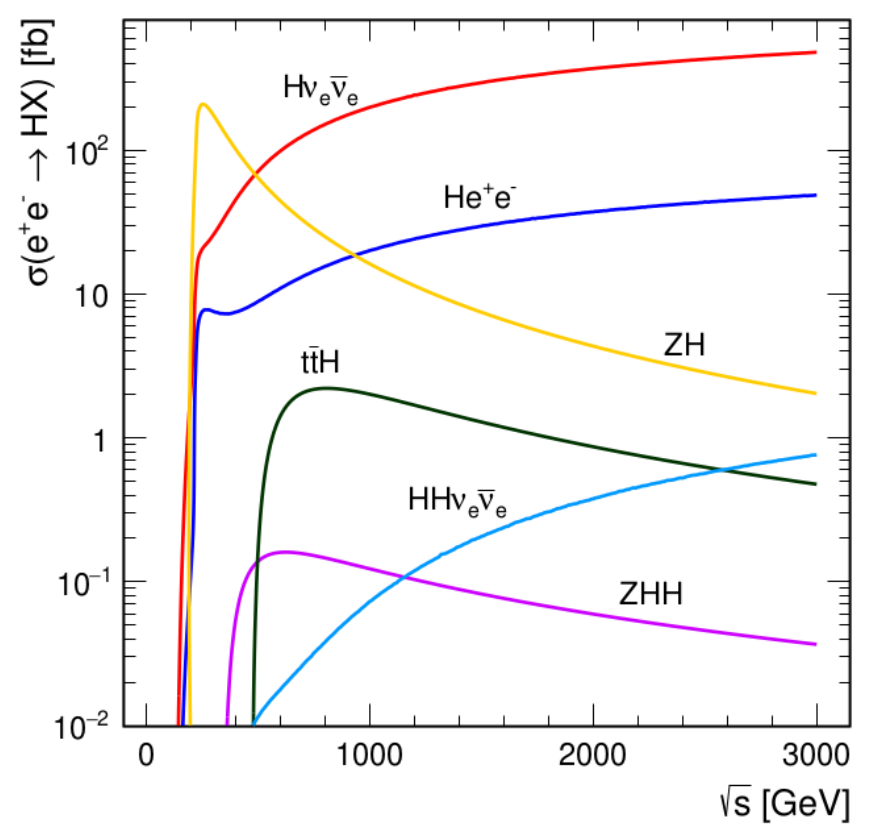

Figure 1: Higgs production cross-sections (figure from [2]).

Linear electron-positron colliders such as the ILC have the potential to fully explore these different processes, thanks to the ability to upgrade the collision energy in a straight-forward way. The ILC will begin at a collision energy of $250 \mathrm{GeV}$, and is designed to allow energy upgrades to at least $1 \mathrm{TeV}$ using the same accelerating technology. The ILC run scenario currently used in physics studies assumes $2000 \mathrm{fb}^{-1}$ of integrated luminosity at $250 \mathrm{GeV}, 200 \mathrm{fb}^{-1}$ at $350 \mathrm{GeV}$, and $4000 \mathrm{fb}^{-1}$ at $500 \mathrm{GeV}$. Linear colliders can provide longitudinally polarised beams; the ILC design foresees electron (positron) beam polarisation of $80 \%$ (30\%). Data will be collected with different 
combinations of left- and right-polarised beams, enabling explicit studies of the chiral nature of particle physics and powerful checks of systematic experimental effects.

The ILC will have a single interaction point (IP) at which collisions occur. The IP can be shared between two detectors, which can be rolled in and out of the beamline using a "push-pull" system. Two experimental groups, SiD and ILD, are developing detector designs for the ILC. These proceedings outline how the requirements in detector performance for the various subsystems of the ILC detectors arise from the Higgs measurements which ILC is designed to make.

\section{Tracking requirements}

A key to model-independent measurements of the Higgs boson at $e^{+} e^{-}$colliders is the Higgsstrahlung process, in which the Higgs is produced together with a Z. The well-defined initial 4-momentum and the measured $\mathrm{Z} 4$-momentum allow the 4-momentum of the recoiling Higgs to be calculated. Particularly useful is the "recoil mass", as shown in fig. 2.
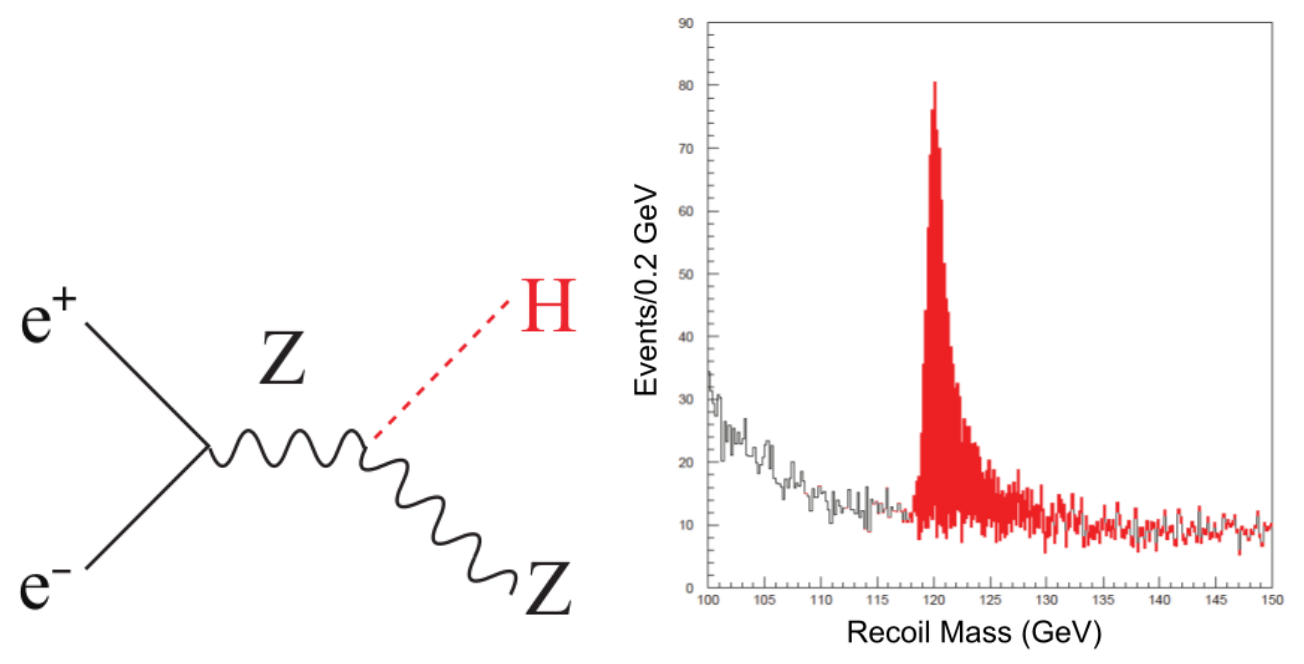

Figure 2: Left: the Higgs-strahlung process. Right: reconstructed mass recoiling against a pair of muons, in full simulation of the $\mathrm{SiD}$ concept [3].

The attainable precision in the recoil mass depends on two factors: the spectrum of the initial state energy \& momentum, and the precision with which the $\mathrm{Z}$ is measured. The initial energy spread results from the accelerator design (a nominal beam energy spread of around $0.2 \%$ ) as well as from beamstrahlung, in which beam particles lose energy as they interact with the strongly-focused opposing beam bunch before the hard collision. The $\mathrm{Z}$ momentum is most precisely measured in its decay to muons, in which the muon momenta are measured by the main tracking system. The momentum resolution of this system should be such that it does not dominate over the effect of the beam energy spread; the momentum resolution does not need to be much better than this, since it will give no practical advantage. A resolution on the transverse momentum $p_{T}$ with an asymptotic value at high $p_{T}$ of $\sigma_{p T} / p_{T}=$ a few $\times 10^{-5} p_{T}$ satisfies this requirement.

The ILD and SiD groups have developed different approaches to the tracker design. ILD has a large gaseous TPC providing up to 220 measurements per track, complemented by inner and outer 
silicon tracker layers to provide high precision measurements at the track start and end. SiD on the other hand uses a silicon-only main tracker providing five high-precision measurements equally spaced along the track, in addition to the hits from the vertex and forward tracking systems. Both of these approaches can attain the required momentum resolution.

\section{Vertexing}

The vertexing abilities of the detector will allow Higgs decays into $b \bar{b}, c \bar{c}$, and $g g$ to be distinguished, enabling these Higgs couplings to be measured. The key is to very accurately measure particle trajectories near the interaction point. This ability is governed by the distance between the IP and the first layer of vertex detector, the position resolution of the vertex detector measurement, and the amount of material contributing to multiple scattering.

The distance to the IP is limited by the distribution of beamstrahlung backgrounds: low energy electrons and positrons produced as the highly focused beam bunches pass through each other. These are constrained by the detector's solenoidal magnetic field, but none the less require that the first vertex detector layer is situated at least $15 \mathrm{~mm}$ from the interaction point. Silicon pixel detectors will provide few-micron point resolution, while thinned sensors, low mass mechanical support, and low power consumption (thanks to "power-pulsing", in which power to the front-end electronics is turned off in the period between bunch trains) and therefore minimal cooling infrastructure will allow a material budget of $0.3 \%$ of a radiation length per vertex detector layer.
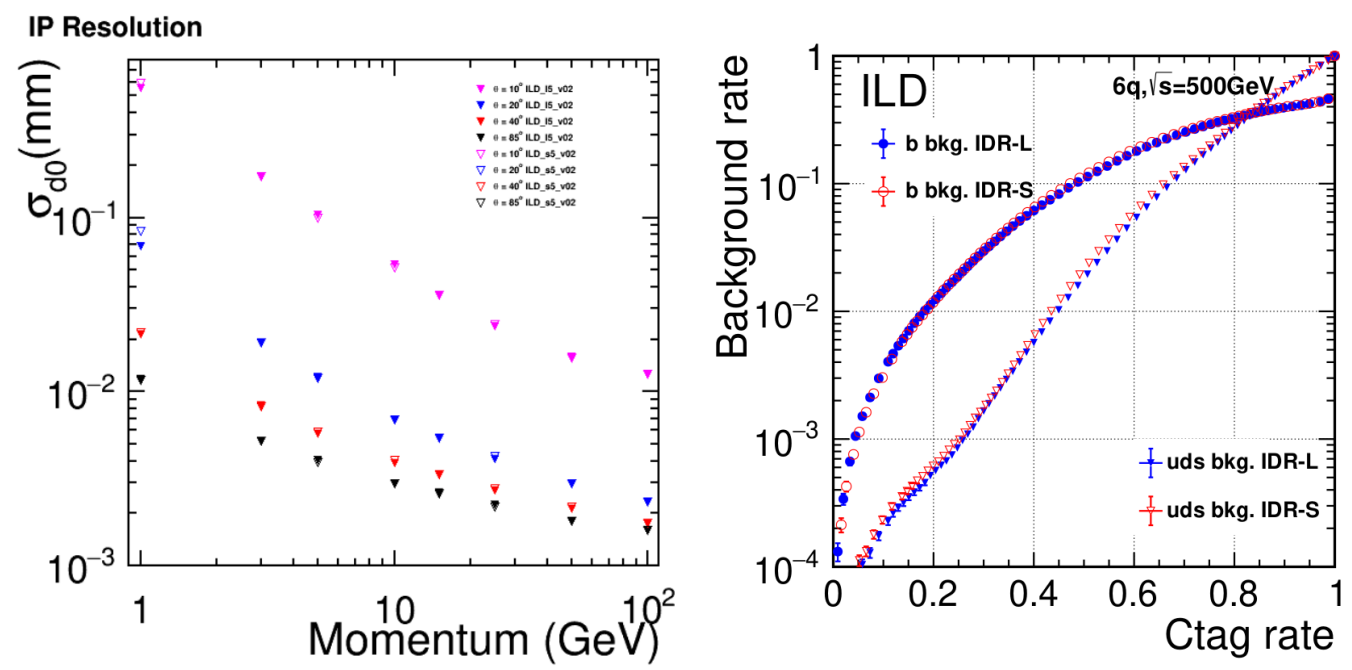

Figure 3: Left: Impact parameter resolution; [black, red, blue, magenta] markers correspond to tracks at a polar angle of $\left[85^{\circ}, 40^{\circ}, 30^{\circ}, 20^{\circ}\right]$, respectively. Right: c-tag performance in ILD [4].

The resulting impact parameter resolution of such designs is shown in fig. 3, which reaches the micron-level for relatively central particles with momentum of at least a few $\mathrm{GeV}$. This performance results in highly efficient identification of $b$ jets and also allows the tagging of charm-jets, as shown in the same figure, resulting in measurements of the the couplings of the Higgs to bottom quarks, charm quarks, and gluons with precisions of $0.60 \%, 1.2 \%$, and $0.96 \%$, respectively [5]. 


\section{Calorimetry}

Calorimetry for ILC detectors is designed around the need to precisely measure hadronic jets. The essential requirement is to have sufficiently good di-jet invariant mass resolution to allow hadronic decays of the Z, W, and Higgs bosons to be distinguished. This will, in turn, allow final states involving the dominant hadronic decays of these bosons to be maximally exploited.

The approach towards achieving this jet energy resolution taken by the ILC detector groups is based on Particle Flow Reconstruction, in which each particle is individually reconstructed in all subdetectors. In particular, the calorimeters should individually measure each particle within hadronic jets, rather than integrating over a collection of nearby particles. This will then allow the much higher precision momentum measurement from the tracking detectors to be used to estimate the charged energy within a jet, and the less precise calorimeters to be used only in the estimation of neutral particles' energy. Given the large average fraction of charged energy within jets $(\sim 65 \%)$, this approach can result in an excellent jet energy measurement.

Calorimeters with high readout granularity, of the order of $\mathrm{cm}^{3}$, are needed to do this successfully at the ILC, resulting in calorimeters with tens of millions of readout channels. This is a practical proposition thanks to advanced technologies such as SiPMs and dedicated low-power ASICs.

Full simulation studies show that jet energy measurement with precision between 3 and 5\% over a wide range of energies is possible with such an approach. As an example, this allows hadronic $\mathrm{Z}$ decays in the Higgs-strahlung process to be used to search for invisible decays of the Higgs, with an expected ILC limit on the invisible decay width of a few parts per mille.
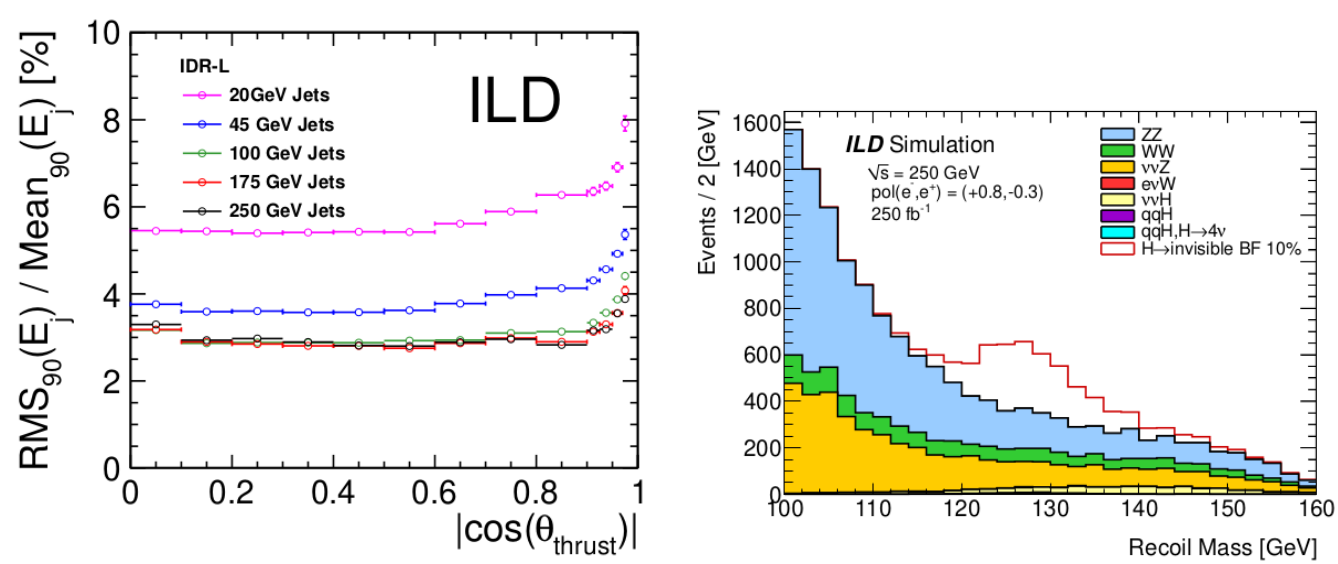

Figure 4: Left: Jet Energy Resolution in ILD full simulation [4]. Right: Hadronic recoil mass in search for invisibly decaying Higgs particles [6].

\section{Conclusion}

The detectors being designed for use at the ILC will consist of highly precise vertex detectors close to the interaction point, light-weight tracking systems for momentum measurement, and highly granular calorimeters for particle flow reconstruction. An almost-totally hermetic detector 
is an additional important design feature. These features, together with the clean experimental environment of electron-positron collisions, will enable the ILC to cover a wide range of physics studies, including searches for new particles, especially those with signatures difficult to identify at the LHC, and precision measurements of QCD, electroweak, and top quark physics. Central to the physics program is the precision measurement of the Higgs sector, which has the potential to show us the way beyond the Standard Model of particle physics.

\section{References}

[1] H. Baer et al., "The International Linear Collider Technical Design Report - Volume 2: Physics," arXiv:1306.6352.

[2] H. Abramowicz et al., "Higgs physics at the CLIC electron-positron linear collider," Eur.Phys.J.C 77 (2017) 7, 475.

[3] T. Behnke et al., "The International Linear Collider Technical Design Report - Volume 4: Detectors," arXiv:1306.6329.

[4] H. Abramowicz et al., "International Large Detector: Interim Design Report,” 2003.01116.

[5] P. Bambade et al., "The International Linear Collider: A Global Project," arXiv:1903.01629.

[6] A. Ishikawa, "Search for invisible decays of the Higgs boson at the ILC,", arXiv:1909.07537. 